\title{
Influence of boiler size and location on one-dimensional two-phase vertical pipe flow
}

\author{
Antigoni Kleanthous ${ }^{a, b}$ and Robert A. Van Gorder ${ }^{a *}$ \\ ${ }^{a}$ Mathematical Institute, University of Oxford, Andrew Wiles Building, Radcliffe Observatory Quarter, Woodstock Road, Oxford OX2 6GG United Kingdom \\ ${ }^{b}$ Department of Mathematics, University College London, Gower Street, London WC1E 6BT United Kingdom \\ *Robert.VanGorder@maths.ox.ac.uk
}

\begin{abstract}
We study the evolution of flow and temperature of a fluid moving upstream in a long, thin vertical pipe when a boiler element is involved. The main goal of this work is to understand how the size and position of the boiler will affect the flow and temperature in the pipe over time, as current literature considers cases where the heater or boiler covers the whole length of the pipe, or when already boiling fluid enters a pipe without a boiler. Therefore, we shall allow for a boiling element which covers only a fraction of the pipe when devising out mathematical model. The boiling process results in a transition to different multiphase flow regimes, and we therefore consider a two-phase flow model. From this model, we obtained a simplified one-dimensional model, since we are concerned with a long, thin pipe, under reasonable assumptions and reductions which still preserve the desired physics. We performed a stability analysis for the boiling boundary denoting the phase change in this model. We then obtained numerical simulations for the steady and transient solutions. The numerical results suggest that both the size and position of the boiler strongly affect the flow regime. In particular, depending on the size of the boiler, transition to other phases might not always occur, and depending on its position along the pipe, the fluid coming out at the top of the pipe might not have the desired thermal profile. As such, one may tailor the position and size of the boiler element in order to obtain a useful thermal profile for particular applications. Such results are of possible relevance in industrial applications where heating or boiling of fluid is required.
\end{abstract}

Keywords: two-phase pipe flow, phase transitions, boiling flow, Stefan problem

\section{Introduction}

Multiphase flow is defined as any fluid flow consisting of more than one phase or component [1]. There are different stages that the multiphase flow takes when moving upwards along a pipe with some boiling action, called flow regimes. When evaporation begins, small gas bubbles begin to form near the walls of the pipe, which then detach and move along the liquid continuum. Such flow regimes are called bubbly flows [2]. When the spacing between the bubbles is large enough so that no collisions occur, we have the so called dispersed bubbly flow, while if collisions are frequent we have dense bubbly flow [3]. As the gas flow increases, the size of the bubbles increases and the flow regime is called slug flow [2]. The gas has the form of large Taylor bubbles, which have a bullet shape [3], and they are separated by liquid slugs. Smaller gas bubbles may also be present in the liquid slug [2]. As the gas flow rate increases further, the liquid slugs are penetrated by the gas [3], and as a result the slugs lose their structure [2], they fragment, and their contents fall [3]. The liquid is then lifted by the gas and the whole procedure repeats. This is the churn flow regime and is known to be chaotic [3]. For very high gas flows, the annular flow regime is observed. In this case, the gas and liquid flow as separate continuous phases [3] with the liquid forming a film at the pipe wall, surrounding the gas flow [2]. As evaporation continues, the liquid film eventually evaporates and a gas disperse flow is observed with small liquid droplets [2]. Disperse flows are defined as flows which consist of finite particles, drops or bubbles (the disperse phase) distributed in a volume of the continuous phase [1]. In general vertical two-phase flow regimes tend to be axisymmetric as the effect of gravity is parallel to the motion [4]. If any other forces exist that are not perpendicular to the flow direction then they might affect the axisymmetry of the flow [4]. The description of these flow regimes is not exhaustive, in fact there are eighty four different flow regimes suggested in literature [4], but not all apply to vertical upwards flow.

Depending on parameter values, some regimes might not be possible. An important parameter is the size of the diameter of the pipe $[3,4,5]$ which affects the existence or non-existence of some flow regimes. For example, Taitel et al. [5] suggested that bubbly flow can exist in small pipes only at high liquid rates. A detailed description of the conditions for transition to a different regime in upward gas-liquid flow in vertical pipes is given by Taitel et al. [5] and Rouhani et al. [4]. Boundaries and external conditions, such as the wall roughness, wall heat flux and flow acceleration, might also affect the flow regimes [4]. One of the most important effects of wall heat flux is dryout, where the liquid loses contact with the wall and as a result the local frictional pressure falls dramatically [4]. A reversed annular flow (a two-phase core is surrounded by a layer of steam in contact with the wall) is observed in this case [4].

There are three ways that multiphase flows can be studied: experimentally, theoretically through mathematical mod- 


\section{Nomenclature}

$\begin{array}{ll}\alpha & \text { gas volume fraction } \\ \beta & \text { liquid volume fraction } \\ \Gamma & \text { Rate of change of phase due to boiling } \\ \rho_{g} & \text { density of the gas phase } \\ \rho_{l} & \text { density of the liquid phase } \\ A & \text { cross-sectional area of the tube } \\ a & \text { Height at which the boiler starts } \\ b & \text { Height at which the boiler ends } \\ C_{p g} & \text { Specific Heat of the gas } \\ C_{p l} & \text { Specific heat of the liquid } \\ E_{g} & \text { Energy transport to the gas phase } \\ E_{l} & \text { Interfacial Energy transport to the liquid phase } \\ F_{g i} & \text { Interfacial stress on the gas phase } \\ F_{g w} & \text { Wall stress on the gas phase } \\ F_{l i} & \text { Interfacial stress on the liquid phase } \\ F_{l w} & \text { Wall Stress on the liquid phase } \\ f_{l w} & \text { Friction factor from the wall } \\ F_{m e t e r} & \text { force from the meter } \\ h_{g} & \text { enthalpy of the gas phase } \\ h_{l} & \text { enthalpy of the liquid }\end{array}$

$H_{g i} \quad$ Heat transfer coefficient of the gas phase

$h_{g i} \quad$ Average interfacial enthalpy of the gas phase

$H_{l i} \quad$ Heat transfer coefficient of the liquid phase

$h_{l i} \quad$ Average interfacial enthalpy of the liquid phase

$L \quad$ Length of the pipe

$p \quad$ pressure

$p_{\text {out }} \quad$ pressure at the top of the pipe

$R \quad$ Ideal gas constant

$r(t) \quad$ Boiling boundary

$s(t) \quad$ Superheat boundary

$T_{g} \quad$ Temperature of the gas phase

$T_{l} \quad$ Temperature of the liquid phase

$T_{g i} \quad$ Interfacial temperature of the gas phase

$T_{l i} \quad$ Interfacial temperature of the liquid phase

$T_{\text {sat }} \quad$ Saturation temperature

$u \quad$ velocity of the liquid phase

$u_{0} \quad$ initial velocity of the liquid phase

$v \quad$ velocity of the gas phase els, and through numerical simulations [1]. There are limitations however to how much experimental studies can resemble reality, and therefore proper mathematical models have to be derived that can be studied either theoretically or computationally [1]. There are different types of models that can be used to study two-phase flows, such as homogeneous, drift-flux, twofluid [2], and slip flow models, each with its own limitations [6]. The drift-flux model is suitable in cases where gravity has a great effect such as flooding and flow reversal [6]. The slip flow model is suitable for high speed uni-directional flow [6]. The most important characteristics of two-phase flow is the presence of interfaces between each phase and the existence of moving boundaries between the different regimes which requires constitutive relations [6]. Each phase can be modelled separately, but having a multi-boundary problem makes it mathematically difficult or impossible to obtain analytical results [6]. The best approach to model a two-phase flow is through averaging, which essentially eliminates unwanted parameters [6]. There are three main types of averaging: Eulerian, Lagrangian, and Boltzmann statistical averages [6].

One goal for studying multiphase flows is to understand under what conditions instabilities might occur. There are different kinds of instabilities, depending on the system studied [2]. For example, a dryout, as mentioned earlier, might lead to failure (burnout or corrosion) of the system especially when this happens in a periodic manner [2]. Different types of instabilities exist such as quasi-static instabilities, concentration waves and dynamic instabilities although one can argue whether concentration waves can be characterised as instabilities [1]. In static-instabilities one considers phenomena that happen on a small time scale, such as transition to annular flow [7] under specific assumptions. Examples of quasi-static instabilities include the turbomachine surge [8,9] observed in compressors, fans, or pumps [1], Ledinegg instability [10] which usually occurs in coffee percolators [1], and Geyser instability [1].

Multiphase flow and boiling processes in pipes continues to 
be an active area of work in the literature $[11,12,13,14]$. There have also been studies on how one might best orient or design pipes for boiling flows $[15,16]$, and also studies on how applied heating at the walls of pipe or other enclosures will modify the properties of such flows [17]. Generally, studies on boiling flow within a pipe fall either into the category of already boiling flows or the category of flows within a pipe with a single large boiler along the entire length of the pipe which generates the multiphase flow through applied heating at the surface of the pipe. Another option is to modify the region of the pipe on which there is a boiler. In many practical applications, particularly for long and thin pipes, one will find it excessive or inconvenient to have a boiler along the entire pipe length. However, to the best of our knowledge, there has been no systematic study of what will happen when only a fractional boiler is applied along a partial boundary around a long, thin pipe.

In the present paper, we are interested in developing a model for a cylindrical pipe that is heated with a boiler on the boundary in some region (not, in general, the entire pipe length), in order to better understand the thermal processes at play. Applications might include coffee makers $[18,19]$, nuclear reactors, refrigerator systems [3], and thermosyphons [20]. Despite the potential applications, we did not find a study of two-phase vertical pipe flow due to a boiler over a segment of the pipe in the literature. As such, we are motivated to consider this problem. In Section 2, we obtain a one-dimensional model for the twophase pile flow, under the assumption that the primary change in flow and thermal variables occurs with height. In order to better understand the influence of the various parameters used in this model, in Section 3 we rigorously non-dimensionalise the model. This non-dimensionalisation allows us to compare the relative importance of various terms, and keeping the essential terms we reduce our model to a more tractable "simplified" model which retains the qualitatively influential terms. Due to the boiling of the fluid, there will exist a boiling boundary at which the phase transition occurs, and we can view the model as a Stefan problem. In Section 5 we consider a stability analysis for the boiling boundary, finding that it is neutrally stable (as was previously shown in the case of boiling fluid entering a pipe or a pipe with a boiling element along its extent). In Section 6 we obtain stationary solutions to the simplified problem, while in Section 7 we obtain numerical solutions which allow us to study transient dynamics within the pipe. We then discuss our results in Section 8 .

\section{Full 1D model for the two-phase flow}

As a full three-dimensional model is quite complicated to analyse or even numerically simulate, we simplify it according to the approach of [7]. In particular, we adapt the model presented in [7] for the system of interest in our case involving a boiler of variable length. We consider a vertical tube $0<z<L$ of (negligibly) small cross-section, with a fluid flowing upwards from $z=0$ to $z=L$ as in Figure 1. We assume that the radius of the pipe is much smaller than its length, and therefore only consider motion in the $z$ direction. Therefore, our results are applicable for long, thin pipes or tubes, so that the radius of the

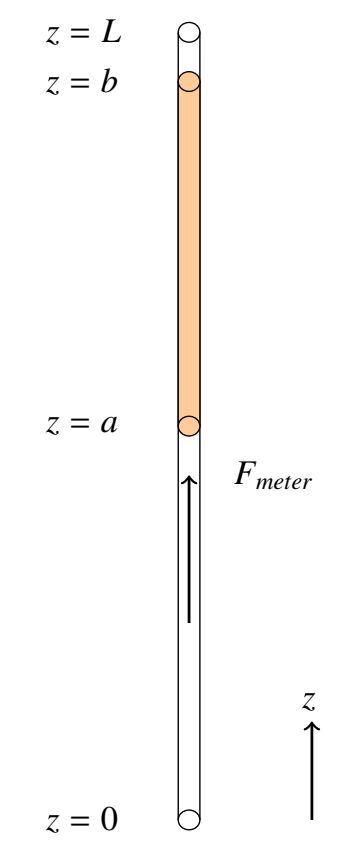

Figure 1: Schematic representation of the system.

pipe is much less than the length of the pipe, $\frac{\text { pipe radius }}{\text { pipe length }}<<1$. For wider pipes, $\frac{\text { pipe radius }}{\text { pipe length }}=O(1)$, and two- or three- dimensional formulations of the model would be required.

The boiler is in the region $a \leq z \leq b$ and since we are considering the $1 \mathrm{D}$ case, we model the action of the boiler by some internal heat source of rate $Q$ (per unit length per unit time). There are two moving boundaries within the boiler, namely $r(t)$, the boiling boundary separating the sub-cooled flow and the two-phase flow, and $s(t)$, the superheat boundary separating the two-phase flow and the superheated flow. Depending on the length of the boiler, not all three regions may be observed within the boiler. Namely, we can have two different cases (assuming that there is always a phase transition):

1. sub-cooled flow before the boiler, sub-cooled flow in the boiler, two-phase flow in the boiler, superheated flow in the boiler and superheated flow after the boiler;

2. sub-cooled flow before the boiler, sub-cooled flow in the boiler, two-phase flow in the boiler and two-phase flow after the boiler.

In the model presented below, the equations have been averaged cross-sectionally and in time [7]. We assume that the cross-sectional area of the tube, $A$, is constant and that the density of the liquid phase $\rho_{l}$ remains constant throughout the motion. Buoyancy effects have been neglected for simplicity, but one can incorporate them for a more complete but complicated model. 


\subsection{Sub-cooled flow below the boiler: $0 \leq z<a$}

We assume that no change in temperature or enthalpy occurs in this region. A suitable model is then

$$
\begin{aligned}
\frac{\partial \rho_{l}}{\partial t}+\frac{\partial}{\partial z}\left(\rho_{l} u\right) & =0 \\
\rho_{l}\left(\frac{\partial u}{\partial t}+u \frac{\partial u}{\partial z}\right) & =-\frac{\partial p}{\partial z}-F_{l w}-\rho_{l} g+F_{\text {meter }} \\
h_{l} & =\bar{h}_{l}
\end{aligned}
$$

where $\rho_{l}$ is the density of the liquid, $u$ the velocity of the liquid, $p$ is the pressure and $h_{l}$ is the enthalpy of the liquid which is assumed to have some constant value $\bar{h}_{l}$. The force from the meter per unit volume is represented by $F_{\text {meter }}$. The term $F_{l w}$ is the friction from the wall and is given by

$$
F_{l w}=\frac{2}{d} f_{l w} \rho_{l}|u| u
$$

where $f_{l w}$ is the friction factor. Now, taking into consideration the assumption that the liquid density is constant, the equations become

$$
\begin{aligned}
u & =u(t) \\
h_{l} & =\bar{h}_{l} \\
\rho_{l} \frac{d u}{d t} & =-\frac{\partial p}{\partial z}-\frac{2}{d} f_{l w} \rho_{l}|u| u-\rho_{l} g+F_{\text {meter }}
\end{aligned}
$$

with initial condition $u(0)=u_{0}$ and boundary condition $p(t, 0)=p_{\text {out }}+\Delta p$.

\subsection{Sub-cooled flow in the boiler: $a \leq z<r(t)$}

In this case, there is no force from the meter, but we have to take into consideration the action of the boiler. Therefore, the equations become

$$
\begin{aligned}
\frac{\partial \rho_{l}}{\partial t}+\frac{\partial}{\partial z}\left(\rho_{l} u\right) & =0 \\
\rho_{l}\left(\frac{\partial u}{\partial t}+u \frac{\partial u}{\partial z}\right) & =-\frac{\partial p}{\partial z}-F_{l w}-\rho_{l} g \\
\rho_{l}\left(\frac{\partial h_{l}}{\partial t}+u \frac{\partial h_{l}}{\partial z}\right) & =\frac{Q}{A}
\end{aligned}
$$

where $F_{l w}$ is as in the previous region. After using the fact that $\rho_{l}$ is constant, we obtain

$$
\begin{aligned}
u & =u(t), \\
\rho_{l} \frac{d u}{d t} & =-\frac{\partial p}{\partial z}-\frac{2}{d} f_{l w} \rho_{l}|u| u-\rho_{l} g, \\
\rho_{l}\left(\frac{\partial h_{l}}{\partial t}+u \frac{\partial h_{l}}{\partial z}\right) & =\frac{Q}{A} .
\end{aligned}
$$

We assume that the variables are continuous between the two regions and therefore impose $p\left(t, a^{+}\right)=p\left(t, a^{-}\right)$and $h\left(t, a^{+}\right)=$ $\bar{h}_{l}$.

\subsection{Two-phase flow in the boiler: $r(t) \leq z<s(t) \leq b$}

The two-phase flow will usually evolve through some or all of the different phase described earlier until it reaches annular flow. The annular flow is usually longer than the other regions before (bubbly flow, slug flow, etc.) and with much larger fluid velocities, so we focus on this flow regime [7]. The conservation of mass equations are

$$
\begin{gathered}
\frac{\partial}{\partial t}\left(\alpha \rho_{g}\right)+\frac{\partial}{\partial z}\left(\alpha \rho_{g} v\right)=\Gamma, \\
\frac{\partial}{\partial t}\left(\beta \rho_{l}\right)+\frac{\partial}{\partial z}\left(\beta \rho_{l} u\right)=-\Gamma .
\end{gathered}
$$

The parameters $\alpha$ and $\beta$ represent the gas and liquid volume fractions, respectively, such that $\alpha+\beta=1$, and $\Gamma$ is the rate of change of phase with time due to the boiling. The variables $\rho_{g}$ and $v$ represent the density and velocity of the gas phase respectively. Conservation of momentum gives

$$
\begin{gathered}
\frac{\partial}{\partial t}\left(\alpha \rho_{g} v\right)+\frac{\partial}{\partial z}\left(\alpha \rho_{g} v^{2}\right)=-\alpha \frac{\partial p}{\partial z}+F_{g i}-F_{g w}-\alpha \rho_{g} g \\
\frac{\partial}{\partial t}\left(\beta \rho_{l} u\right)+\frac{\partial}{\partial z}\left(\beta \rho_{l} u^{2}\right)=-\beta \frac{\partial p}{\partial z}-F_{l w}+F_{l i}-\beta \rho_{l} g
\end{gathered}
$$

where $F_{l w}$ is the wall stress on the liquid phase, $F_{l i}$ the interfacial stress on the liquid, $F_{g i}$ the interfacial stress on the gas such that $F_{l i}=-F_{g i}$ and $F_{g w}$ is the wall stress on the gas phase. Since we are considering annular flow and there is no contact between the wall and the gas, we have $F_{g w}=0$. The interfacial stress is given by

$$
F_{l i}=\frac{2}{d} f_{l i} \rho_{g}|v-\chi u|(v-\chi u),
$$

where $\chi=2$ by [21]. In the equations of momentum conservation, it was assumed that various profile coefficients during the averaging are equal to one and also that the pressures in the liquid and gas phase are the same. This is realistic, but creates problems when $\rho_{g}=\rho_{l}=1$ and $\Gamma=F_{l w}=F_{l i}=0$ as the equations become ill-posed [7]. Any other parameters are thought to be small and therefore neglected. A complete model for the 1D problem can be found in [2], on which this simplified version [7] was based. Equations for conservation of enthalpy are

$$
\begin{aligned}
\alpha \rho_{g}\left(\frac{\partial h_{g}}{\partial t}+v \frac{\partial h_{g}}{\partial z}\right) & =\Gamma\left(h_{g i}-h_{g}\right)+E_{g}, \\
\beta \rho_{l}\left(\frac{\partial h_{l}}{\partial t}+u \frac{\partial h_{l}}{\partial z}\right) & =\Gamma\left(h_{l}-h_{l i}\right)+E_{l}+\frac{Q}{A} .
\end{aligned}
$$

The variables $h_{l}$ and $h_{g}$ represent the liquid and gas enthalpy respectively, while $E_{l}$ and $E_{g}$ are the intefacial energy transports to the liquid and gas phase respectively. The parameters $h_{l i}$ and $h_{g i}$ are the average inerfacial enthalpies. In addition to that, we need the averaged interfacial Stefan condition

$$
E_{l}+E_{g}+\Gamma\left(h_{g i}-h_{l i}\right)=\frac{Q}{A} .
$$

The interfacial heat transports are given by

$$
E_{l}=\frac{H_{l i}\left(T_{l i}-T_{l}\right)}{L_{s}}, E_{g}=\frac{H_{g i}\left(T_{g i}-T_{g}\right)}{L_{s}},
$$


where $H_{l i}$ and $H_{g i}$ are the heat transfer coefficients, $T_{l}$ is the temperature of the liquid and $T_{g}$ the temperature of the gas. The parameters $T_{g i}$ and $T_{l i}$ are the interfacial temperatures of gas and liquid respectively and are assumed to be constant and equal to $T_{\text {sat }}$, the local saturation temperature. We ignore any variations of $T_{\text {sat }}$ with pressure and therefore obtain that the interfacial enthalpies are constant

$$
h_{g i}=h_{g 0}, \quad h_{l i}=h_{l 0} \text {. }
$$

Therefore the enthalpies can be defined by

$$
h_{l}=h_{l 0}+C_{p l}\left(T_{l}-T_{s a t}\right), \quad h_{g}=h_{g 0}+C_{p g}\left(T_{g}-T_{s a t}\right),
$$

where $C_{p l}$ and $C_{p g}$ are the specific heats of the liquid and gas respectively. The parameter $1 / L_{S}$ is the average surface area per unit volume. For annular flow [7]

$$
\frac{1}{L_{s}}=\frac{4 \sqrt{\alpha}}{d} .
$$

Finally, we need another equation for $\rho_{g}$ since we assume it is not constant. We take the ideal gas law

$$
\rho_{g}=\frac{p}{R T_{g}},
$$

where $R$ is the ideal gas constant.

We again assume that the variables remain continuous between the subcooled flow and the two-phase flow and therefore require

$$
\begin{aligned}
p\left(t, r^{-}(t)\right) & =p\left(t, r^{+}(t)\right), \\
u\left(t, r^{-}(t)\right) & =u\left(t, r^{+}(t)\right), \\
h_{l}\left(t, r^{-}(t)\right) & =h_{l}\left(t, r^{+}(t)\right),
\end{aligned}
$$

while the rest of the variables require boundary conditions on $r(t)$ such that

$$
\beta(t, r(t))=1, \quad \alpha(t, r(t))=0, \quad \Gamma(t, r(t))=0,
$$

and suitable boundary conditions for $\rho_{g}$ and $v$.

\subsection{Superheated flow in the boiler: $s(t) \leq z<b$}

In this region, all the liquid has transformed to gas and therefore the equations are

$$
\begin{aligned}
\frac{\partial \rho_{g}}{\partial t}+\frac{\partial}{\partial z}\left(\rho_{g} v\right) & =0 \\
\frac{\partial}{\partial t}\left(\rho_{g} v\right)+\frac{\partial}{\partial z}\left(\rho_{g} v^{2}\right) & =-\frac{\partial p}{\partial z}-F_{g w}-\rho_{g} g \\
\rho_{g}\left(\frac{\partial h_{g}}{\partial t}+v \frac{\partial h_{g}}{\partial z}\right) & =\frac{Q}{A},
\end{aligned}
$$

where

$$
F_{g w}=\frac{2}{d} f_{g w} \rho_{g}|v| v
$$

supplemented by the equation of state

$$
\rho_{g}=\frac{p}{R T_{g}} .
$$

Assuming that the variables remain continuous across the two regions separated by the boundary $s(t)$ we have

$$
\begin{aligned}
& \rho_{g}\left(t, r^{-}(t)\right)=\rho_{g}\left(t, r^{+}(t)\right), \quad v\left(t, r^{-}(t)\right)=v\left(t, r^{+}(t)\right), \\
& h_{g}\left(t, r^{-}(t)\right)=h_{g}\left(t, r^{+}(t)\right), \quad p\left(t, r^{-}(t)\right)=p\left(t, r^{+}(t)\right) .
\end{aligned}
$$

\subsection{Superheated flow above the boiler: $b \leq z \leq L$}

Equations in this case are

$$
\begin{aligned}
\frac{\partial \rho_{g}}{\partial t}+\frac{\partial}{\partial z}\left(\rho_{g} v\right) & =0 \\
\frac{\partial}{\partial t}\left(\rho_{g} v\right)+\frac{\partial}{\partial z}\left(\rho_{g} v^{2}\right) & =-\frac{\partial p}{\partial z}-F_{g w}-\rho_{g} g \\
\frac{\partial h_{g}}{\partial t}+v \frac{\partial h_{g}}{\partial z} & =0
\end{aligned}
$$

where

$$
F_{g w}=\frac{2}{d} f_{g w} \rho_{g}|v| v
$$

supplement by the equation of state

$$
\rho_{g}=\frac{p}{R T_{g}} .
$$

Assuming that the variables remain continuous across the two regions separated by the boundary $b$ we have

$$
\begin{aligned}
\rho_{g}\left(t, b^{-}\right) & =\rho_{g}\left(t, b^{+}\right), \\
v\left(t, b^{-}\right) & =v\left(t, b^{+}\right), \\
h_{g}\left(t, b^{-}\right) & =h_{g}\left(t, b^{+}\right), \\
p\left(t, b^{-}\right) & =p\left(t, b^{+}\right),
\end{aligned}
$$

along with the boundary condition $p(t, L)=p_{\text {out }}$.

\subsection{Two-phase flow above the boiler: $b \leq z \leq L$}

This is the case when there is no superheated flow within the pipe, and therefore the two-phase flow continues after the fluid exits the boiler. In this case we have

$$
\begin{gathered}
\frac{\partial}{\partial t}\left(\alpha \rho_{g}\right)+\frac{\partial}{\partial z}\left(\alpha \rho_{g} v\right)=\Gamma, \\
\frac{\partial}{\partial t}\left(\beta \rho_{l}\right)+\frac{\partial}{\partial z}\left(\beta \rho_{l} u\right)=-\Gamma, \\
\frac{\partial}{\partial t}\left(\alpha \rho_{g} v\right)+\frac{\partial}{\partial z}\left(\alpha \rho_{g} v^{2}\right)=-\alpha \frac{\partial p}{\partial z}-F_{g i}-\alpha \rho_{g} g, \\
\frac{\partial}{\partial t}\left(\beta \rho_{l} u\right)+\frac{\partial}{\partial z}\left(\beta \rho_{l} u^{2}\right)=-\beta \frac{\partial p}{\partial z}-F_{l w}-F_{l i}-\beta \rho_{l} g, \\
\alpha \rho_{g}\left(\frac{\partial h_{g}}{\partial t}+v \frac{\partial h_{g}}{\partial z}\right)=\Gamma\left(h_{g}-h_{g i}\right)+E_{g}, \\
\beta \rho_{l}\left(\frac{\partial h_{l}}{\partial t}+u \frac{\partial h_{l}}{\partial z}\right)=\Gamma\left(h_{l}-h_{l i}\right)+E_{l},
\end{gathered}
$$

along with the Stefan condition

$$
E_{l}+E_{g}+\Gamma\left(h_{g i}-h_{l i}\right)=0
$$

and equation of state

$$
\rho_{g}=\frac{p}{R T_{g}} .
$$

Again we assume continuity across $b$, along with the boundary condition $p(t, L)=p_{\text {out }}$. 


\section{Non-dimensionalization of the full $1 D$ model}

We choose similar non-dimensional parameters as in [7]. The relevant scales were chosen by [7] so that the dimensionless variables are $O(1)$. The velocities in the two-phase region are expected to be large, and therefore the parameters were chosen so that they balance the two-phase equations. We define the non-dimensional variables as $\beta=\epsilon \beta^{*}, v=V v^{*}, u=U u^{*}, p=$ $p_{\text {out }}+P p^{*}, \Gamma=\bar{\Gamma} \gamma, \rho_{g}=\bar{\rho}_{g} \rho_{g}^{*}, h_{g}=h_{g 0}+C_{p g} \Delta T h_{g}^{*}, h_{l}=$ $h_{l 0}+C_{p l} \Delta T h_{l}^{*}, z=L z^{*}, t=\frac{L}{U} t^{*}$, where $\Delta T=T_{\text {sat }}-T_{0}, \bar{\rho}_{g} V=$ $L \bar{\Gamma}, \rho_{l} \epsilon U=L \bar{\Gamma}, \bar{\rho}_{g}=\frac{p_{\text {out }}}{R T_{\text {sat }}}, f_{l w} \rho_{l} U^{2}=f_{l i} \bar{\rho}_{g} V^{2}, P=\Delta p=$ $\frac{2}{d} L f_{l w} \rho_{l} U^{2}$. We further define the non-dimensional parameters

$$
\begin{gathered}
\epsilon=\left(\frac{f_{l w} \bar{\rho}_{g}}{f_{l i} \rho_{l}}\right)^{1 / 2}, a_{1}=\frac{U}{V}, \epsilon_{2}=\frac{\epsilon d}{2 f_{l w} L}, \epsilon_{3}=\frac{d}{2 L f_{l i}}, \\
a_{4}=\frac{4 H_{g i} \Delta T}{d \bar{\Gamma}\left(h_{g 0}-h_{l 0}\right)}, a_{5}=\frac{C_{p l} \Delta T}{h_{g 0}-h_{l 0}}, a_{5 b}=\frac{C_{p g} \Delta T}{h_{g 0}-h_{l 0}}, \\
\epsilon_{6}=\frac{d \bar{\Gamma}\left(h_{g 0}-h_{l 0}\right)}{4 H_{l i} \Delta T}, a_{7}=\frac{d C_{p g} \bar{\Gamma}}{4 H_{g i}}, a_{8}=\frac{P}{p_{\text {out }}}, a_{9}=\frac{\Delta T}{T_{\text {sat }}}, \\
\epsilon_{10}=\frac{d}{2 L f_{l w}}, a_{11}=\frac{d g}{2 f_{l w} U^{2}}, a_{12}=\frac{d g}{2 f_{l i} V^{2}}, q=\frac{Q}{A \bar{\Gamma}\left(h_{g 0}-h_{l 0}\right)} .
\end{gathered}
$$

3.1. Subcooled flow below the boiler: $0 \leq z^{*}<a^{*}=\frac{a}{L}$

The equations for the velocity and enthalpy are given by

$$
u^{*}=u^{*}(t), h_{l}^{*}=\bar{h}_{l}^{*},
$$

where $\bar{h}_{l}^{*}$ is defined such that

$$
\bar{h}_{l}^{*}=\frac{\bar{h}_{l}-h_{l 0}}{C_{p l} \Delta T},
$$

and the equation for the pressure becomes

$$
\epsilon_{10} \frac{d u^{*}}{d t^{*}}=-\frac{\partial p^{*}}{\partial z^{*}}-\left(u^{*}\right)^{2}-a_{11}+F_{m e t e r}^{*},
$$

where $F_{\text {meter }}^{*}$ takes the form

$$
F_{\text {meter }}^{*}=\frac{d}{2 f_{l w} \rho_{l} U^{2}} F_{\text {meter }} .
$$

Therefore, we have

$$
\begin{aligned}
u^{*} & =u^{*}(t), \\
h_{l}^{*} & =\bar{h}_{l}^{*}, \\
\epsilon_{10} \frac{d u^{*}}{d t^{*}} & =-\frac{\partial p^{*}}{\partial z^{*}}-\left(u^{*}\right)^{2}-a_{11}+F_{\text {meter }}^{*},
\end{aligned}
$$

with initial condition $u^{*}(0)=u_{0}^{*}$ and boundary condition $p^{*}\left(t^{*}, 0\right)=1$.

\subsection{Sub-cooled flow in the boiler: $a^{*} \leq z^{*}<r^{*}\left(t^{*}\right)$}

The equations become

$$
\begin{aligned}
u^{*} & =u^{*}\left(t^{*}\right), \\
\epsilon_{10} \frac{d u^{*}}{d t^{*}} & =-\frac{\partial p^{*}}{\partial z^{*}}-\left(u^{*}\right)^{2}-a_{11}, \\
\frac{\partial h_{l}^{*}}{\partial t^{*}}+u^{*} \frac{\partial h_{l}^{*}}{\partial z^{*}} & =\frac{\epsilon q}{a_{5}},
\end{aligned}
$$

with continuity of all variables across the boundary $a^{*}$. The boiling boundary $r^{*}\left(t^{*}\right)$ can be found by solving $h_{l}^{*}\left(t^{*}, r^{*}\left(t^{*}\right)\right)=$ 0 .
3.3. Two-phase flow in the boiler: $r^{*}\left(t^{*}\right) \leq z^{*}<s^{*}\left(t^{*}\right) \leq b^{*}$

Conservation of mass equations become

$$
\begin{aligned}
a_{1} \frac{\partial}{\partial t^{*}}\left(\left(1-\epsilon \beta^{*}\right) \rho_{g}^{*}\right)+\frac{\partial}{\partial z}\left(\left(1-\epsilon \beta^{*}\right) \rho_{g}^{*} v^{*}\right) & =\gamma, \\
\frac{\partial \beta^{*}}{\partial t^{*}}+\frac{\partial}{\partial z^{*}}\left(\beta^{*} u^{*}\right) & =-\gamma,
\end{aligned}
$$

and conservation of momentum equations become

$$
\begin{gathered}
\epsilon_{3}\left[a_{1} \frac{\partial}{\partial t^{*}}\left(\left(1-\epsilon \beta^{*}\right) \rho_{g}^{*} v^{*}\right)+\frac{\partial}{\partial z^{*}}\left(\left(1-\epsilon \beta^{*}\right) \rho_{g}^{*} v^{* 2}\right)\right] \\
=-\left(1-\epsilon \beta^{*}\right) \frac{\partial p^{*}}{\partial z^{*}}-\rho_{g}^{*}\left(v^{*}-\chi a_{1} u^{*}\right)^{2}-a_{12}\left(1-\epsilon \beta^{*}\right) \rho_{g}^{*} \\
\epsilon_{2}\left[\frac{\partial}{\partial t^{*}}\left(\beta^{*} u^{2 *}\right)+\frac{\partial}{\partial z^{*}}\left(\beta^{*} u^{* 2}\right)\right] \\
=-\epsilon \beta^{*} \frac{\partial p^{*}}{\partial z^{*}}-u^{2 *}+\rho_{g}^{*}\left(v^{*}-\chi a_{1} u^{*}\right)^{2}-\epsilon \beta^{*} a_{11}
\end{gathered}
$$

The equations for conservation of enthalpy become

$$
\begin{aligned}
& a_{7}\left(1-\epsilon \beta^{*}\right) \rho_{g}^{*}\left[a_{1} \frac{\partial h_{g}^{*}}{\partial t^{*}}+v^{*} \frac{\partial h_{g}^{*}}{\partial z^{*}}\right]=-\left[a_{7} \gamma+\left(1-\epsilon \beta^{*}\right)^{1 / 2}\right] h_{g}^{*}, \\
& a_{5} \epsilon_{6} \beta^{*}\left[\frac{\partial h_{l}^{*}}{\partial t^{*}}+u^{*} \frac{\partial h_{l}^{*}}{\partial z^{*}}\right]=\epsilon_{6} q+\left[\gamma a_{5} \epsilon_{6}-\left(1-\epsilon \beta^{*}\right)^{1 / 2}\right] h_{l}^{*},
\end{aligned}
$$

with the ideal gas law

$$
\rho_{g}^{*}=\frac{1+a_{8} p^{*}}{1+a_{9} h_{g}^{*}},
$$

and the Stefan Condition

$$
\gamma=q+a_{4}\left(1-\epsilon \beta^{*}\right)^{1 / 2} h_{g}^{*}+\frac{1}{\epsilon_{6}}\left(1-\epsilon \beta^{*}\right)^{1 / 2} h_{l}^{*} .
$$

Again, we assume continuity across the boundary $r^{*}\left(t^{*}\right)$ and also $\beta^{*}\left(t^{*}, r^{*}\left(t^{*}\right)\right)=1 / \epsilon$. The superheat boundary $s^{*}\left(t^{*}\right)$ can be found by solving $\beta^{*}\left(t^{*}, s^{*}\left(t^{*}\right)\right)=0$.

\subsection{Superheated flow in the boiler: $s^{*}\left(t^{*}\right) \leq z^{*}<b^{*}$}

The equations are

$$
\begin{gathered}
a_{1} \frac{\partial \rho_{g}^{*}}{\partial t^{*}}+\frac{\partial}{\partial z^{*}}\left(\rho_{g}^{*} v^{*}\right)=0 \\
\epsilon_{3}\left[a_{1} \frac{\partial}{\partial t^{*}}\left(\rho_{g}^{*} v^{*}\right)+\frac{\partial}{\partial z^{*}}\left(\rho_{g}^{*} v^{* 2}\right)\right]=-\frac{\partial p^{*}}{\partial z^{*}}-\frac{f_{g w}}{f_{l i}} \rho_{g}^{*} v^{* 2}-a_{12} \rho_{g}^{*} \\
\rho_{g}^{*}\left[a_{1} \frac{\partial h_{g}^{*}}{\partial t^{*}}+v^{*} \frac{\partial h_{g}^{*}}{\partial z^{*}}\right]=\frac{q}{a_{5 b}}
\end{gathered}
$$

along with the equation of state

$$
\rho_{g}^{*}=\frac{1+a_{8} p^{*}}{1+a_{9} h_{g}^{*}},
$$

and continuity across the superheat boundary $s^{*}\left(t^{*}\right)$. 
3.5. Superheated flow above the boiler: $b^{*} \leq z^{*} \leq 1$

The equations in this case become

$$
\begin{gathered}
a_{1} \frac{\partial \rho_{g}^{*}}{\partial t^{*}}+\frac{\partial}{\partial z^{*}}\left(\rho_{g}^{*} v^{*}\right)=0 \\
\epsilon_{3}\left[a_{1} \frac{\partial}{\partial t^{*}}\left(\rho_{g}^{*} v^{*}\right)+\frac{\partial}{\partial z^{*}}\left(\rho_{g}^{*} v^{* 2}\right)\right]=-\frac{\partial p^{*}}{\partial z^{*}}-\frac{f_{g w}}{f_{l i}} \rho_{g}^{*} v^{* 2}-a_{12} \rho_{g}^{*} \\
\rho_{g}^{*}\left[a_{1} \frac{\partial h_{g}^{*}}{\partial t^{*}}+v^{*} \frac{\partial h_{g}^{*}}{\partial z^{*}}\right]=0
\end{gathered}
$$

along with the equation of state

$$
\rho_{g}^{*}=\frac{1+a_{8} p^{*}}{1+a_{9} h_{g}^{*}},
$$

and continuity across the superheat boundary $b^{*}$. Furthermore, we set the boundary condition $p^{*}\left(t^{*}, L^{*}\right)=0$.

3.6. Two-phase flow above the boiler: $b^{*} \leq z^{*} \leq 1$

The equations in this case become

$$
\begin{gathered}
a_{1} \frac{\partial}{\partial t^{*}}\left(\left(1-\epsilon \beta^{*}\right) \rho_{g}^{*}\right)+\frac{\partial}{\partial z^{*}}\left(\left(1-\epsilon \beta^{*}\right) \rho_{g}^{*} v^{*}\right)=\gamma \\
\frac{\beta^{*}}{\partial t^{*}}+\frac{\partial}{\partial z^{*}}\left(\beta^{*} u^{*}\right)=\gamma \\
\epsilon_{3}\left[a_{1} \frac{\partial}{\partial t^{*}}\left(\left(1-\epsilon \beta^{*}\right) \rho_{g}^{*} v^{*}\right)+\frac{\partial}{\partial z^{*}}\left(\left(1-\epsilon \beta^{*}\right) \rho_{g}^{*} v^{*}\right)\right] \\
=-\left(1-\epsilon \beta^{*}\right) \frac{\partial p^{*}}{\partial z^{*}}-\rho_{g}^{*}\left(v^{*}-\chi a_{1} u^{*}\right)^{2}-a_{12}\left(1-\epsilon \beta^{*}\right) \rho_{g}^{*} \\
\epsilon_{2}\left[\frac{\partial}{\partial t^{*}}\left(\beta^{*} u^{*}\right)+\frac{\partial}{\partial z^{*}}\left(\beta^{*} u^{* 2}\right)\right] \\
=-\epsilon \beta^{*} \frac{\partial p^{*}}{\partial z^{*}}-u^{*}+\rho_{g}^{*}\left(v^{*}-\chi a_{1} u^{*}\right)^{2}-\epsilon \beta^{*} a_{11} \\
a_{7}\left(1-\epsilon \beta^{*}\right) \rho_{g}^{*}\left[a_{1} \frac{\partial h_{g}^{*}}{\partial t^{*}}+v^{*} \frac{\partial h_{g}^{*}}{\partial z^{*}}\right]=-\left[a_{7} \gamma+\left(1-\epsilon \beta^{*}\right)^{1 / 2}\right] h_{g}^{*} \\
a_{5} \epsilon_{6} \beta^{*}\left[\frac{\partial h_{l}^{*}}{\partial t^{*}}+u^{*} \frac{\partial h_{l}^{*}}{\partial z^{*}}\right]=\left[\gamma a_{5} \epsilon \epsilon_{6}-\left(1-\epsilon \beta^{*}\right)^{1 / 2}\right] h_{l}^{*}
\end{gathered}
$$

with equation of state

$$
\rho_{g}^{*}=\frac{1+a_{8} p^{*}}{1+a_{9} h_{g}^{*}},
$$

and the Stefan Condition

$$
\gamma=a_{4}\left(1-\epsilon \beta^{*}\right)^{1 / 2} h_{g}^{*}+\frac{1}{\epsilon_{6}}\left(1-\epsilon \beta^{*}\right)^{1 / 2} h_{l}^{*} .
$$

Again, we assume continuity across the boundary $b^{*}$ and also boundary condition $p^{*}\left(t^{*}, 1\right)=0$.

\subsection{Parameter values}

We now proceed to determine the size of different parameters, and non-dimensional numbers. The parameter values can be seen in Table 1. The non-dimensional parameters are then found to be $\epsilon=0.09, a_{1}=0.44, \epsilon_{2}=0.009, \epsilon_{3}=0.2, a_{4}=6$, $a_{5}=0.76, \epsilon_{6}=0.01, a_{7}=0.13, a_{8}=0.5, a_{9}=0.5, \epsilon_{10}=0.1$, $a_{11}=0.01, a_{12}=0.0004$. We shall use these values in the forthcoming analysis and simulations of the model.

\begin{tabular}{ll} 
Parameter & Value \\
\hline$\rho_{l}$ & $760 \mathrm{~kg} / \mathrm{m}^{3}$ \\
$\bar{\rho}_{g}$ & $30 \mathrm{~kg} / \mathrm{m}^{3}$ \\
$C_{p l}$ & $5.2 \times 10^{3} \mathrm{~J} / \mathrm{kgK}$ \\
$C_{p g}$ & $4.6 \times 10^{3} \mathrm{~J} / \mathrm{kgK}$ \\
$T_{s a t}$ & $270 \mathrm{~K}$ \\
$\Delta T$ & $250 \mathrm{~K}$ \\
$p_{\text {out }}$ & $60 \mathrm{bars}$ \\
$f_{l i}$ & 0.02 \\
$f_{l w}$ & 0.004 \\
$h_{l 0}-h_{g 0}$ & $1.6 \times 10^{6} \mathrm{~J} / \mathrm{kg}$ \\
$d$ & $0.014 \mathrm{~m}$ \\
$A$ & $1.54 \times 10^{-4} \mathrm{~m}^{2}$ \\
$L$ & $20 \mathrm{~m}$ \\
$R$ & $8.314 \mathrm{~J} / \mathrm{mol} \mathrm{K}$ \\
$H_{l i}$ & $1.7 \times 10^{5} \mathrm{~W} / \mathrm{m}^{2} \mathrm{~K}$ \\
$H_{g i}$ & $0.7 \times 10^{4} \mathrm{~W} / \mathrm{m}^{2} \mathrm{~K}$
\end{tabular}

Table 1: Parameter values adapted from [2] and [7] for our problem.

\section{Simplified 1D two-phase model}

Since many of the non-dimensional parameters are very small, we proceed with an asymptotic reduction, as was done for the related model studied in [7]. We omit the stars in the non-dimensional model obtained earlier for simplicity.

\subsection{Sub-cooled flow below and in the boiler}

Considering the boiling boundary $r$, we know that $\beta=1 / \epsilon$ on $z=r$ and that $\beta u$ has to be continuous across the boundary. Therefore we need $u \approx \epsilon$ in $z<r$ such that the velocity field of the sub-cooled flow below and in the boiler is given by

$$
u(t)=\epsilon u_{0}(t)
$$

Now, for the asymptotic reduction we will ignore any term that is of order $10^{-2}$ or less. The equation for pressure before the boiler then becomes

$$
\frac{\partial p}{\partial z}=F_{m e t e r}
$$

which can be solved for

$$
p(z)=\int_{0}^{z} F_{\text {meter }}(\theta) d \theta+p_{0}(t)
$$

The enthalpy below the boiler is constant $h_{l}=\bar{h}_{l}$, while in the boiler satisfies

$$
\frac{\partial h_{l}}{\partial t}+\epsilon u_{0}(t) \frac{\partial h_{l}}{\partial z}=\frac{\epsilon q}{a_{5}} .
$$

Transforming the above into slow time $\tau=\epsilon t$ we obtain

$$
\frac{\partial h_{l}}{\partial \tau}+u_{0}(\tau) \frac{\partial h_{l}}{\partial z}=\frac{q}{a_{5}} .
$$

The characteristics of the above satisfy

$$
\frac{d \tau}{d x}=1, \frac{d z}{d x}=u_{0}(\tau), \frac{d h_{l}}{d x}=\frac{q}{a_{5}} .
$$


Solving for $z$ and $\tau$ we have

$$
z=a+\int_{\tau_{a}}^{\tau} u_{0}(\theta) d \theta
$$

and solving for $h_{l}$ in terms of $\tau$

$$
h_{l}(\tau, z)=\bar{h}_{l}+\frac{q}{a_{5}}\left(\tau-\tau_{a}\right) .
$$

Finally, combining the above we obtain the implicit solution

$$
z(\tau)=a+\int_{\tau-\frac{a_{5}}{q}\left(h_{l}(\tau, z)-\bar{h}_{l}\right)}^{\tau} u_{0}(\theta) d \theta
$$

The term

$$
\tau_{s c}=\frac{a_{5}}{q}
$$

represents the sub-cooling delay. The boiling boundary can be found by setting $h_{l}(\tau, r)=0$ such that

$$
r(\tau)=a+\int_{\tau+\tau_{s c} \bar{h}_{l}}^{\tau} u_{0}(\theta) d \theta
$$

Since the boiling boundary $r$ is given in terms of the slow time $\tau$, it will vary slowly with $t$. Taking $\bar{h}_{l}=-1$ we obtain

$$
r(\tau)=a+\int_{\tau-\tau_{s c}}^{\tau} u_{0}(\theta) d \theta .
$$

\subsection{Two-phase flow}

Neglecting very small terms, we obtain the approximate model

$$
\begin{aligned}
a_{1} \frac{\partial \rho_{g}}{\partial t}+\frac{\partial\left(\rho_{g} v\right)}{\partial z} & =\gamma, \\
\frac{\partial \beta}{\partial t}+\frac{\partial(\beta u)}{\partial z} & =-\gamma, \\
a_{7} \rho_{g} v \frac{\partial h_{g}}{\partial z} & =-\left(1+a_{7} \gamma\right) h_{g}, \\
\frac{\partial p}{\partial z} & =-u^{2}, \\
u & =\frac{\sqrt{\rho_{g}}}{1+\chi a_{1} \sqrt{\rho_{g}}} v, \\
h_{l} & =0, \\
\gamma & =q+a_{4} h_{g}, \\
\rho_{g} & =\frac{1+a_{8} p}{1+a_{9} h_{g}} .
\end{aligned}
$$

We remark that, neglecting the non-dimensional numbers $\epsilon_{2}$ and $\epsilon_{3}$ might lead to singularities and discontinuity of $\beta, u$ and $v$ on $z=r$, as pointed out in [7]. If there is no transition to the superheat phase, then the same equations with $q=0$ are used to model the flow above the boiler.

We impose the following boundary conditions on $z=r$ :

$$
\begin{aligned}
p & : \text { continuous, } \\
h_{g} & =0, \\
\rho_{g} & =1+a_{8} p, \\
v & =\frac{1+\chi a_{1} \sqrt{\rho_{g}}}{\sqrt{\rho_{g}}} \epsilon u_{0}, \\
\beta u & =u_{0} .
\end{aligned}
$$

The above imply that $h_{g} \equiv 0, \gamma \equiv q$, and the rest of the variables are determined by

$$
\begin{aligned}
a_{1} \frac{\partial \rho_{g}}{\partial t}+\frac{\partial\left(\rho_{g} v\right)}{\partial z} & =q, \\
\frac{\partial \beta}{\partial t}+\frac{\partial(\beta u)}{\partial z} & =-q, \\
\frac{\partial p}{\partial z} & =-u^{2}, \\
u & =\frac{\sqrt{\rho_{g}}}{1+\chi a_{1} \sqrt{\rho_{g}}} v, \\
\rho_{g} & =1+a_{8} p .
\end{aligned}
$$

Transition to the superheat phase begins as soon as $\beta=0$. If no transition to superheated flow exists, then we also impose $p=0$ at $z=1$.

\subsection{Superheated Flow}

Neglecting again small terms we have

$$
\begin{aligned}
a_{1} \frac{\partial \rho_{g}}{\partial t}+\frac{\partial\left(\rho_{g} v\right)}{\partial z} & =0 \\
\rho_{g}\left(a_{1} \frac{\partial h_{g}}{\partial t}+v \frac{\partial h_{g}}{\partial z}\right) & =\frac{q}{a_{5 b}} \\
\frac{\partial p}{\partial z} & =-\frac{f_{g w}}{f_{l i}} \rho_{g} v^{2} \\
\rho_{g} & =\frac{1+a_{8} p}{1+a_{9} h_{g}} .
\end{aligned}
$$

Since we want continuity over the superheat boundary $z=s$ we impose the following conditions

$p$ : continuous, $v$ : continuous, $\rho_{g}$ : continuous, $h_{g}=0$.

When the gas leaves the boiler, the same equations apply when $q=0$ and continuity of all variables over $z=b$. We further impose the boundary condition $p=0$ at $z=1$.

\section{Stability analysis of the boiling boundary}

We now proceed to find quasi-static solutions as suggested in [7]. As already mentioned above, $r$ varies on the slow time scale $\tau$. To find quasi-static solutions (solutions that vary slowly with time $t$ and therefore any derivatives with respect to time are negligible), we assume no instabilities in the two-phase region. Then the equations for the two -phase flow can give solutions

$$
\begin{aligned}
\rho_{g} v & =q(z-r), \\
\beta u & =u_{0}-q(z-r), \\
\frac{\partial p}{\partial z} & =-u^{2}, \\
u & =\frac{\sqrt{\rho_{g}}}{1+\chi a_{1} \sqrt{\rho_{g}}} v, \\
\rho g & =1+a_{8} p,
\end{aligned}
$$

where $r$ is given by

$$
r(\tau)=a+\int_{\tau-\tau_{s c}}^{\tau} u_{0}(\theta) d \theta .
$$


As Aldridge et al. [7] argue, one can show that the dynamics of the system can be described by

$$
r\left(u_{0}\right)=a+\int_{\tau-\tau_{s c}}^{\tau} u_{0}(\theta) d \theta
$$

when considering the quasi-static solution. Now, for a steady state, all variables are independent of time (both slow and fast), and therefore the steady boiling boundary is given by

$$
r\left(u_{0}\right)=a+\tau_{s c} u_{0}
$$

Considering a perturbation of the steady state

$$
u=u_{0}+\delta u_{1}(\tau)
$$

we obtain

$$
r\left(u_{0}+\delta u_{1}(\tau)\right)=a+\int_{\tau-\tau_{s c}}^{\tau}\left(u_{0}+\delta u_{1}(\theta)\right) d \theta
$$

Using a first order Taylor expansion on the left hand side and integrating the right hand side, the above gives

$$
r\left(u_{0}\right)+\delta u_{1}(\tau) r^{\prime}\left(u_{0}\right)=a+\tau_{s c} u_{0}+\delta \int_{\tau-\tau_{s c}}^{\tau} u_{1}(\theta) d \theta+O\left(\delta^{2}\right) .
$$

Considering $O(1)$ terms we have

$$
r\left(u_{0}\right)=a+\tau_{s c} u_{0}
$$

which is the steady state, and to $O(\delta)$, we have

$$
u_{1}(\tau) r^{\prime}\left(u_{0}\right)=\int_{\tau-\tau_{s c}}^{\tau} u_{1}(\theta) d \theta
$$

which if differentiated once with respect to $\tau$ gives

$$
u_{1}^{\prime}(\tau) r^{\prime}\left(u_{0}\right)=u_{1}(\tau)-u_{1}\left(\tau-\tau_{s c}\right)
$$

This is a delay differential equation. Assuming $u_{1}=e^{\lambda \tau}$ for some parameter $\lambda$ to be determined, we have

$$
\lambda r^{\prime}\left(u_{0}\right)=1-e^{-\lambda \tau_{s c}} .
$$

Noticing that $r^{\prime}\left(u_{0}\right)=\tau_{s c}$, the above can be rewritten in the form

$$
\left(\lambda \tau_{s c}-1\right) e^{\lambda \tau_{s c}-1}=-\frac{1}{e}
$$

which has solution

$$
\lambda \tau_{s c}-1=W\left(-\frac{1}{e}\right)
$$

where $W$ is the Lambert $W$ function. This has the solution $\lambda \tau_{s c}-$ $1=-1$, which gives that either $\lambda=0$ or $\tau_{s c}=0$, with the latter implying that the boiling boundary lies at the beginning of the boiler at $r=a$. Since $\tau_{s c}=0$ would not be realistic we have that $\lambda=0$ which implies that the steady state is neutrally stable.

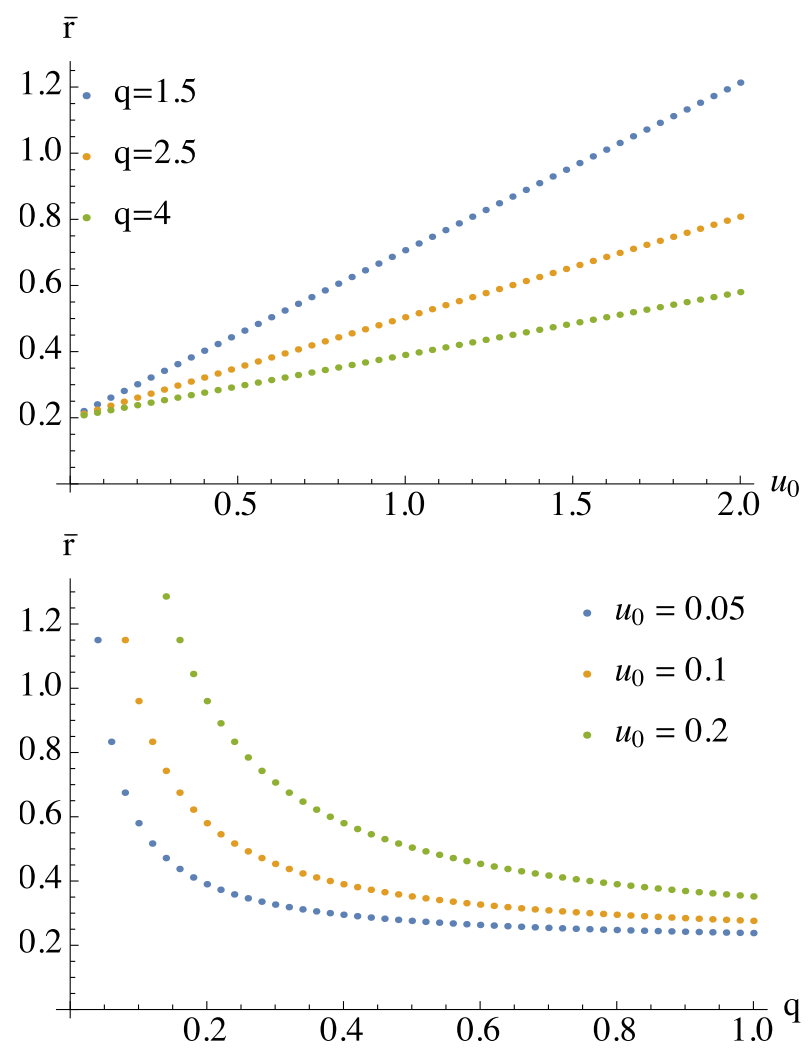

Figure 2: Boiling boundary $\bar{r}$ against inlet velocity $u_{0}$ (top) and heat strength $q$ (bottom).

\section{Steady solutions of the simplified 1D model}

We now obtain steady solutions and therefore all variables depend only on $z$. Further, the boiling and superheat boundaries are also constants, $\bar{r}$ and $\bar{s}$, respectively. The steady states for the sub-cooled flow can be found analytically but for the two-phase flow it is easier to work numerically, and so we find all steady states for every phase numerically using the function NDSolve in Mathematica. The non-dimensional numbers were taken as in Table 1. We take the length of the pipe $L=1$ and put the boiler in the region $0.2 \leq z \leq 0.8$. The inlet velocity is assumed to be $u_{0}=1$ and the pressure $p_{0}=1$. Note that we have assumed the meter force to be zero for simplicity.

We examine how the boiling boundary depends on the inlet velocity $u_{0}$ and the heat strength $q$. The results can be seen in Figure 2. We fix different values of $q$ for the first graph and different values for $u_{0}$ for the second. The observations agree with the analytical solution for the steady state obtained in Equation (131). Namely, the boiling boundary $\bar{r}$ shows linear dependence with inlet velocity $u_{0}$ with the intersection point at $a=0.2$. On the other hand, the boiling boundary decreases with increasing $q$, approaching $a=0.2$ asymptotically (recall that $\tau_{s c}=a 5 / q$ ). Of course one has to ignore values of $\bar{r}$ that exceed $L=1$ or even $b=0.8$, as in this case only single-phase flow (sub-cooled flow) would be observed.

In Figure 3 we present the steady states for three different values of heat strength $q$. Notice that $q=1.5$ and $q=2.5$ result in a 

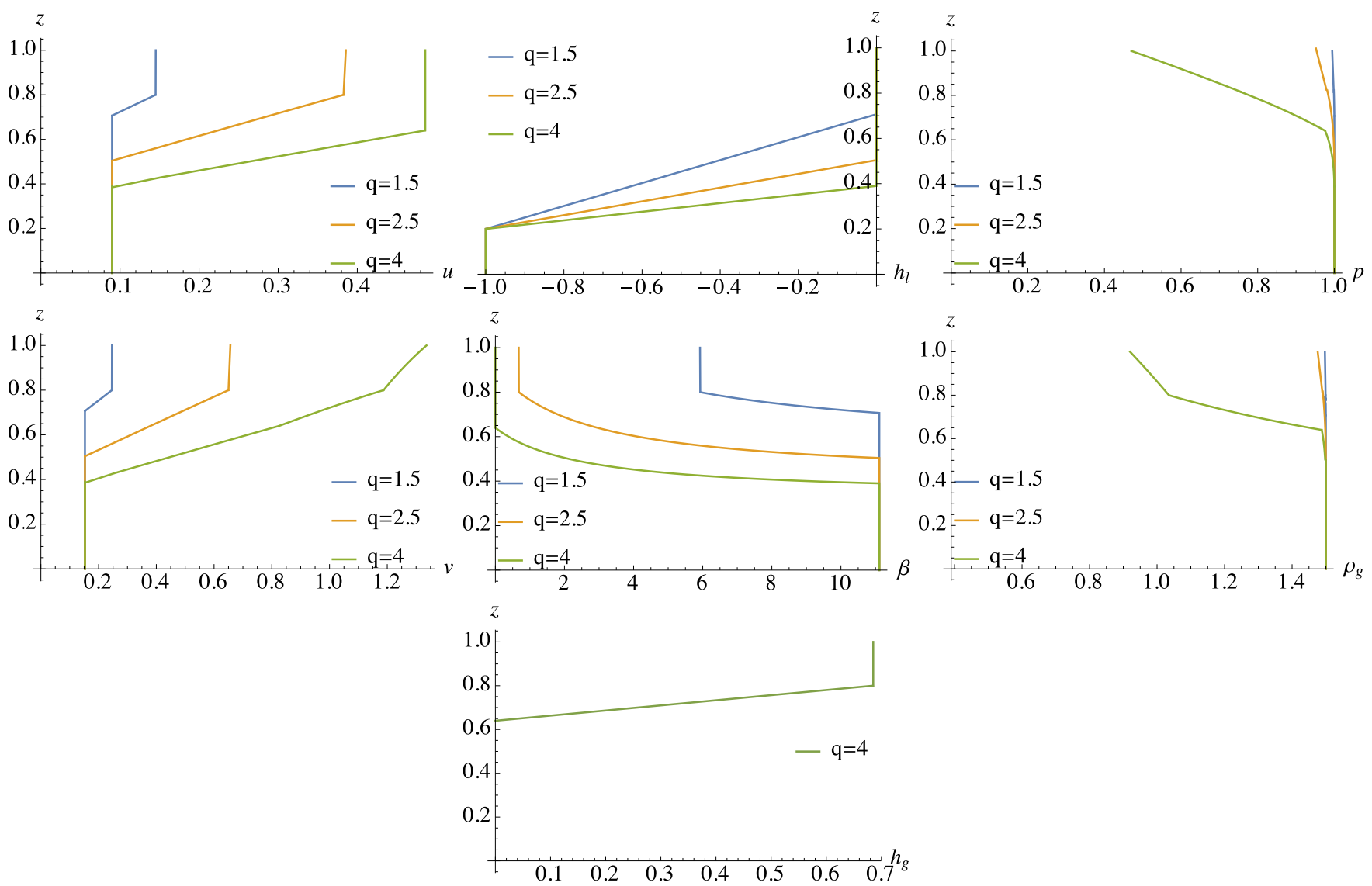

Figure 3: Steady solutions when the boiler lies in $0.2 \leq z \leq 0.8$ with different heating strengths $q$. 
two-phase flow that continues after the boiler, with the boiling boundaries computed at $\bar{r}=0.71$ and $\bar{r}=0.50$, respectively. For $q=4$ a two-phase flow followed by superheated flow in observed, with the boiling boundary computed at $\bar{r}=0.39$ and the superheat boundary at $\bar{s}=0.64$. Notice that the graph for the gas enthalpy $h_{g}$ has only one curve, since in only one case superheated flow is observed. Similar qualitative behaviour is observed in all three cases, except there is a greater pressure and gas density loss during the superheated flow compared to the two-phase flow. We should also note that the variables $v, \beta$ and $\rho_{g}$ do not exist below the boiling boundary $\bar{r}$, but we represent them as constants here corresponding to their boundary value on $\bar{r}$. The same applies to $h_{g}$, which does not exist before superheated flow and that is the reason we present results for $z \geq \bar{s}=0.64$.

\section{Numerical solutions for the simplified 1D model}

We now proceed to solve the asymptotically reduced model numerically. The motion of the fluid before it encounters the boiler is described by

$$
p(t)=p_{0}(t), u(t)=\epsilon u_{0}(t), \quad h_{l}=-1,
$$

for some functions $p_{0}(t)$ and $u_{0}(t)$ that we are free to prescribe (and assuming that the force from the meter is zero). As soon as the fluid enters the boiler at $z=a$, the equation for enthalpy is

$$
\frac{\partial h_{l}}{\partial t}+\frac{\partial}{\partial z}\left(u(t) h_{l}\right)=\frac{\epsilon q}{a_{5}},
$$

which can be solved numerically by the Lax-Friedrichs scheme [22]

$$
\begin{array}{r}
\frac{1}{\Delta t}\left[\left(h_{l}\right)_{r}^{n+1}-\frac{1}{2}\left(\left(h_{l}\right)_{r+1}^{n}+\left(h_{l}\right)_{r-1}^{n}\right)\right] \\
+\frac{u\left(t_{n}\right)}{2 \Delta z}\left[\left(h_{l}\right)_{r+1}^{n}-\left(h_{l}\right)_{r-1}^{n}\right]=\frac{\epsilon q}{a_{5}}
\end{array}
$$

for $\left(h_{l}\right)_{r}^{n+1}$ with the boundary condition $\left(h_{l}\right)_{r_{a}}^{n+1}=-1$. The pressure and velocity are still described by $p(t)=p_{0}(t)$ and $u(t)=\epsilon u_{0}(t)$. As soon as $\left(h_{l}\right)_{r}^{n+1}$ becomes zero or positive and provided that we are still in the region of the boiler, we set $\left(h_{l}\right)_{r}^{n+1}=0$ for the rest of the pipe. Then the motion of the multiphase flow is described by

$$
\begin{aligned}
a_{1} \frac{\partial \rho_{g}}{\partial t}+\frac{\partial\left(\rho_{g} v\right)}{\partial z} & =q, \\
\frac{\partial \beta}{\partial t}+\frac{\partial(\beta u)}{\partial z} & =-q, \\
\frac{\partial p}{\partial z} & =-u^{2}, \\
u & =\frac{\sqrt{\rho_{g}}}{1+\chi a_{1} \sqrt{\rho_{g}}} v, \\
\rho_{g} & =1+a_{8} p .
\end{aligned}
$$

The equation for $\beta$ is a hyperbolic PDE in conservation form that can be solved again by the Lax-Friedrichs Scheme. We can substitute Equation (148) into (144) and solve for the pressure again using the Lax-Friedrichs Scheme, and then update for the density via Equation (148). Once we have the density and pressure we can solve Equations (146) and (147) for the velocity fields. The numerical method appears to be mesh free from the trials we have run.

We assume that at $z=r, p$, and $u$ are continuous and use initial and boundary conditions for the other three variables such that

$$
\beta=\frac{1}{\epsilon}, \quad \rho_{g}=1+a_{8} p, \quad v=\frac{1+\chi a_{1} \sqrt{\rho_{g}}}{\rho_{g}} \epsilon u_{0} .
$$

As soon as the fluid passes $z=b$, the motion is still described with Equations (144) - (148) with $q=0$ and continuity of all variables across $z=b$. We assume there is no superheat flow in the boiler and this can be achieved by choosing the appropriate value of $q$.

The above algorithm is implement in MATLAB. We assume the pipe to be of length $L=1$ with a boiler present in the region $0.2 \leq z \leq 0.8$ in Figure 4 and on the whole pipe in Figure 5. We set the final time to $t_{\text {final }}=1$ and set $p_{0}(t)=1-t$ and $u_{0}(t)=\sqrt{t}$. We take $\Delta t=1 \times 10^{-4}, \Delta z=0.01$ and $q=40$. Initial conditions are $h_{l}=-1, u=0$ and $p=1$ with boundary conditions matching the initial conditions on $z=0$ and zero flux on $z=1$ for $h_{l}$ and $u$, and zero for the pressure $p$. Note that the variables $\beta$, $\rho_{g}$ and $v$ do not actually exist below the boiling boundary and therefore should not show any evolution with time. The results presented here below the boiler are estimates for the initial and boundary conditions so that the code will have the required data to compute those three variables as soon as the two-phase flow begins.

The discontinuity observed in the numerical solutions near $z=0.6$ (the boiling boundary) is probably due to the fact that we ignored $\epsilon_{2}$ and $\epsilon_{3}$ in the reduction of the model, as argued earlier [7]. The variables seem to have a smoother behaviour in the case of the full boiler rather than the case where the boiler takes only a part of the pipe. As expected, the boiling boundary seems to be higher when the pipe is partially heated rather than fully.

In addition to the specific cases shown in Figures 4-5, other cases were considered as well. A third type of solution was found when we considered a boiler of smaller size (for instance, $0.6 \leq z \leq 0.8$ with the same heating intensity parameter $q=40$ ) which admitted solutions that did not have a phasetransition from one- to two-phase flow and we therefore omit the graphs of this case. This is something to consider when applying the results, as this shows that as the size of the boiler affects whether there is transition to a two-phase flow and even a superheated flow.

Note that to check our numerical scheme, we may compare our simulations with those of [7] in the regime where the boiler covers the entire length of the pipe. As our model was reduced in a similar way to the model of [7], such a comparison makes complete sense, and we find that our numerical approach will faithfully reproduce their qualitative findings. Furthermore, as in [7], we find that the boiling boundary is neutrally stable, which is consistent with the numerical simulations. Therefore, 

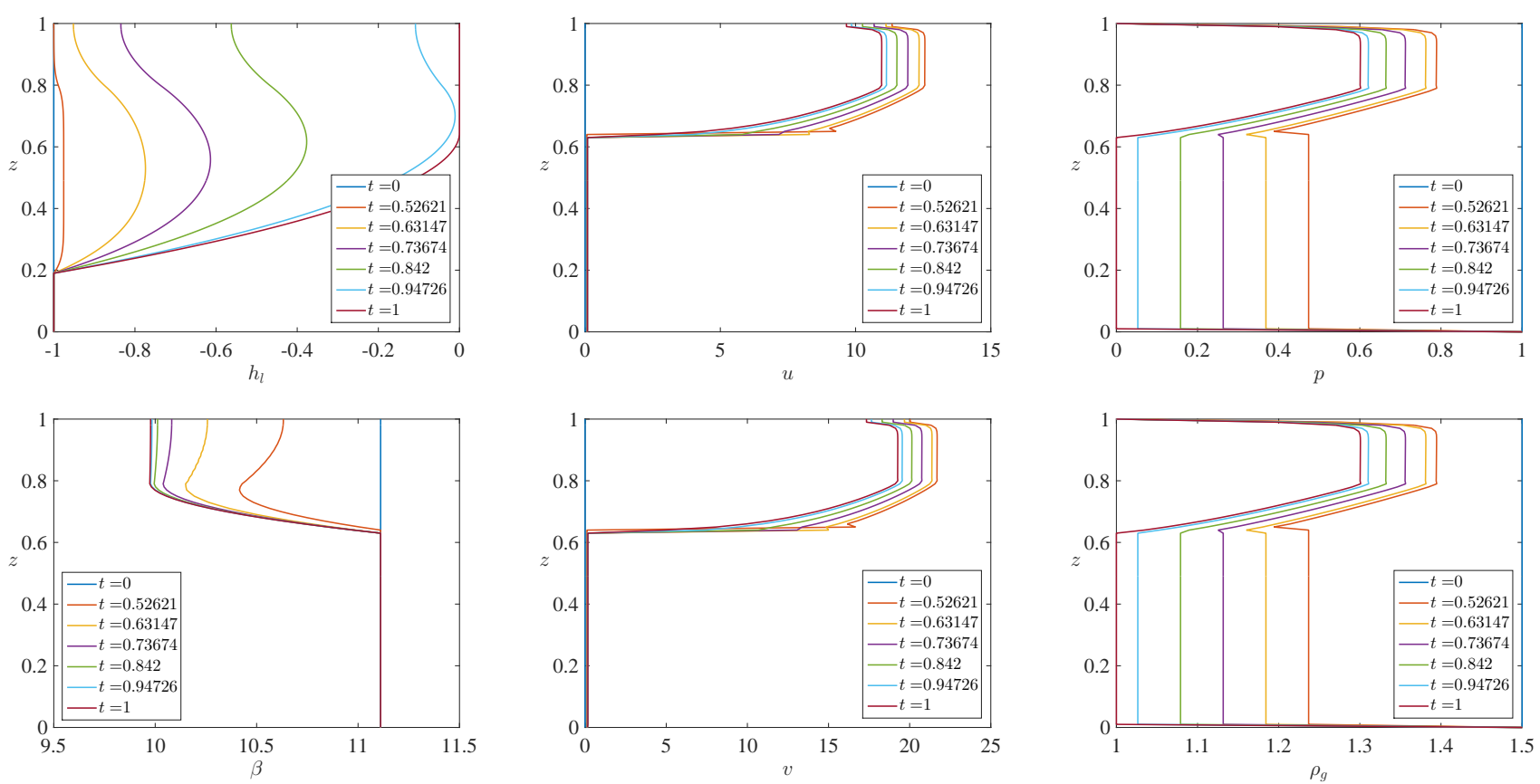

Figure 4: Numerical simulation with $p_{0}(t)=1-t, u_{0}(t)=\sqrt{t}$ with the boiler present in the region $0.2 \leq z \leq 0.8$. The boiler has strength $q=40$ in this example.
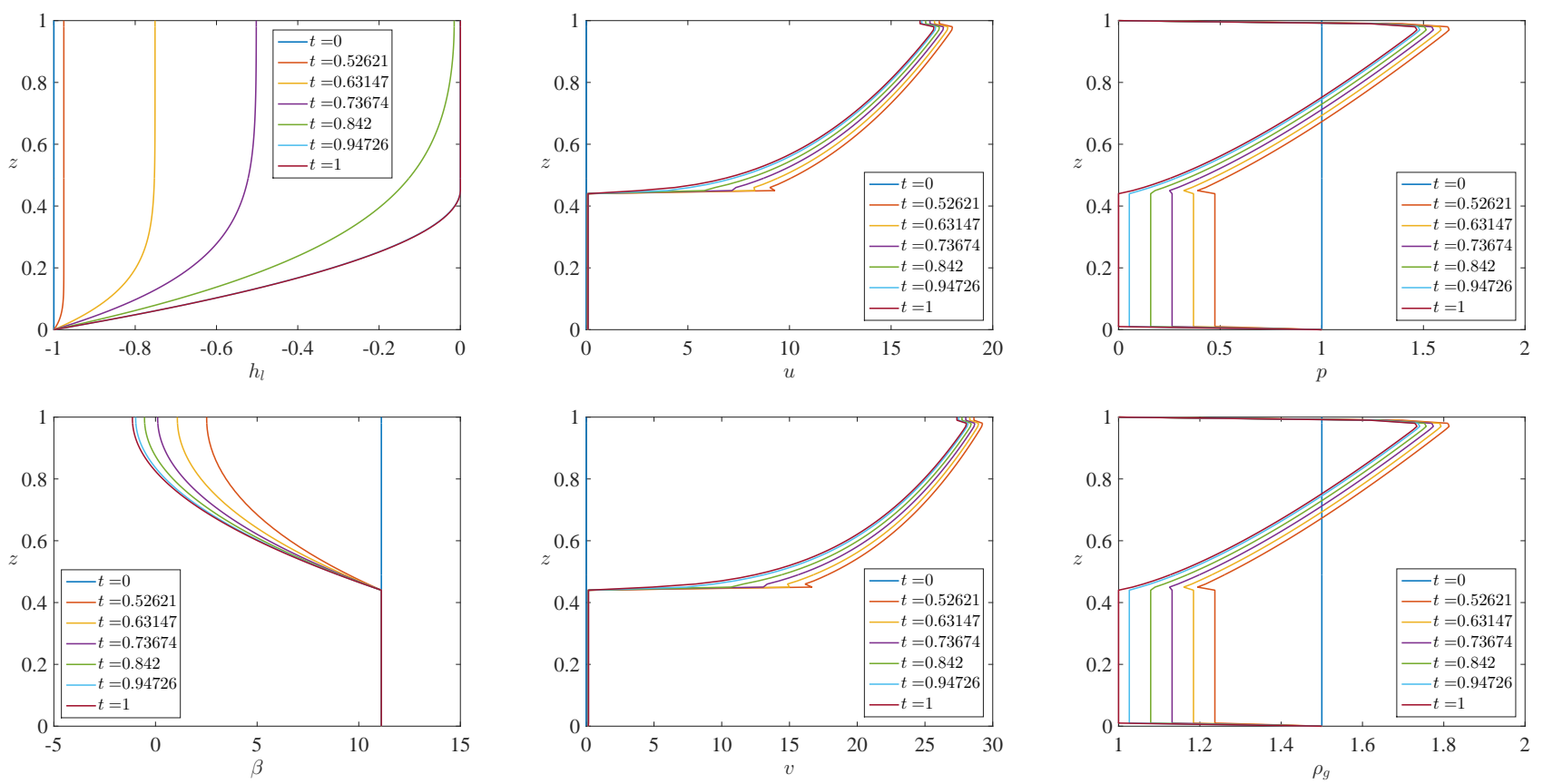

Figure 5: Numerical simulation with $p_{0}(t)=1-t, u_{0}(t)=\sqrt{t}$. The boiler covers the whole pipe $(0 \leq z \leq 1)$ and has strength $q=40$. 
although no direct experimental work is available for comparison, our results do compare favorably with numerical and analytical work studying a pipe which has a boiler over the entire pipe length, $L$, lending validity to the numerical and analytical results we present here.

\section{Discussion}

We have studied the flow and temperature evolution of a fluid flowing upstream a vertical pipe which is heated by a boiler in some region. Under physically reasonable simplifications, we derived a reduced model that assumes flow in one dimension only (height). Following an appropriate nondimensionalization, the reduced model was reduced even further, upon neglecting small dimensionless groups which do not strongly influence the qualitative behavior of the solutions. We performed a stability analysis on the boiling boundary (derived from the reduced model) and then obtained the steady state solutions numerically. The behaviour of the steady boiling boundary with respect to inlet velocity and heat strength of the boiler was studied, and we observed that the higher the inlet velocity, the higher the boiling boundary is, which should be taken into consideration for applications in industry. Greater heat strength resulted in a lower boiling boundary within the pipe which is what one would expect as the fluid would boil sooner (and hence lower in the pipe). Finally, we solved the simplified model numerically in order to deduce the transient behavior of the solutions. In these numerical simulations, we considered the cases where the boiler covers only part of the pipe, as well as the case where the boiler covers the whole pipe (which was the only case previously considered in literature). The results for the second case seemed to have a smoother behaviour than the first case, as the fluid more gradually heats rather than suddenly heating when it encounters the sub-region with a boiler. Therefore, a full boiler covering the entire pipe might be needed for applications where a gradual thermal transition is called for, while applications simply calling for the heating of fluid before it exits the top of a pipe should not be sensitive to this feature, and a smaller boiler region could be used. Another case with a very small partial boiler was considered, and for this cases we did not observe transition to boiling and the resulting twophase flow, as the fluid did not have time to heat sufficiently before reaching the top of the pipe. We reach the conclusion that both the size and position of the boiler strongly affect the flow regimes and temperature distribution.

These results could be taken into consideration in industrial applications, depending on the desired outcome. The partial boiler could be used in cases where a boiling effect is required in the pipe, but steady velocity or pressure are required above the boiler as in Figure 4. The case of the partial boiler is probably more economic as the boiler is not working on the full length of the pipe, rather on some section of it. Depending on the desired outcome, a partial boiler could give the desired result using less power. The partial boiler can also be used from some point until the end of the pipe, if the desired outcome on the top of the pipe is as in Figure 5. Indeed, if one simply wishes to get the fluid to boiling before it exits the top of the pipe, then a partial boiler is sufficient and a full boiler covering the entire pipe may be a waste. Of course, these simulations pertain to very specific examples. To match such results to any particular industrial application, one would need to properly calibrate the model parameters, in order to then obtain an optimal profile for the boiler size, position, and heating intensity $(q)$.

Regarding future work, the full one-dimensional problem could be studied in greater depth either numerically or analytically, although we suspect that qualitatively the results will be quite similar to what we obtain for the simplified onedimensional problem. Furthermore, the numerical solution of the full model is somewhat tricky, as it consists of hyperbolic PDEs that are not in conservation form, which can lead to numerical instabilities when the system is discretized. The results we obtain suggest some differences do exist based on the size and position of the boiler, as well as the inlet velocity of the fluid. As this aspect of the boiler will modify the velocity and thermal field within the pipe, the results may be of use to those manufacturers of coffee makers, nuclear reactor cooling systems, refrigerator systems, and thermosyphons, as for such applications one wishes to maintain certain desired heat and mass transfer properties within the pipe flow. If precision calculations are required for such applications, then one may wish to reconsider the full model to make sure any quantitative differences between it and the simplified model are accounted for.

\section{Conclusions}

Using a one-dimensional two-phase model of the heat and fluid flow within a long, thin vertical pipe, we have been able to study the evolution of flow and temperature of a fluid moving upstream in a vertical pipe when a localized boiler element is involved. In particular, we considered the changes in heat and mass transfer cased by changes in the size and position of a partial boiler along the pipe surface, rather than a full boiler covering the entire pipe (as is more common in the literature). A stability analysis for the boiling boundary demonstrated neutral stability, as was true in the case where the boiler covered the entire pipe length, which was previously studied in [7]. Numerical simulations for the steady and transient solutions were then employed to solve the one-dimensional, two-phase model, and we found that both the size and position of the boiler strongly affect the flow regime. This is of relevance to experiments or even applications, as depending on the size of the boiler, transition to other phases might not always occur, and hence there might not be a desired boiling of the fluid. Meanwhile, depending on the position of the boiler along the pipe surface, the fluid coming out at the top of the pipe might not have the desired thermal profile. The results suggest that, when employing a partial boiler, one must be careful to calibrate both the size and the position of the boiler in order to obtain desired heat and mass transfer for use in a specific application.

\section{References}

[1] Brennen, C. E. (2005). Fundamentals of multiphase flow. Cambridge university press.

[2] Aldridge, C. J., \& Fowler, A. C. (1996). Stability and instability in evaporating two-phase flows. Surveys on Mathematics for Industry, 6, 75-107. 
[3] Shaban, H., \& Tavoularis, S. (2014). Identification of flow regime in vertical upward air-water pipe flow using differential pressure signals and elastic maps. International Journal of Multiphase Flow, 61, 62-72.

[4] Rouhani, S. Z., \& Sohal, M. S. (1983). Two-phase flow patterns: A review of research results. Progress in Nuclear Energy, 11(3), 219-259.

[5] Taitel, Y., Barnea, D., \& Dukler, A. E. (1980). Modelling flow pattern transitions for steady upward gas-liquid flow in vertical tubes. AIChE Journal, 26(3), 345-354.

[6] Ishii, M., \& Kocamustafaogullari, G. (1982). Two-phase-flow models and their limitations (No. CONF-820811-3). Argonne National Lab., IL (USA); Wisconsin Univ., Milwaukee (USA).

[7] Aldridge, C. J., \& Fowler, A. C. (1993). Dynamics of a reduced model of two-phase flow in a boiling channel. In Instabilities in Multiphase Flows (pp. 137-148). Springer US.

[8] Emmons, H. W., Pearson, C. E., \& Grant, H. P. (1955). Compressor surge and stall propagation. Trans. ASME, 77(4), 455-469.

[9] Greitzer, E. M. (1981). The stability of pumping systems-the 1980 Freeman Scholar Lecture. Journal of Fluids Engineering, 103(2), 193-242.

[10] Ledinegg, M. (1938). Instability of flow during natural and forced circulation. Die Warme, 61(8), 891-898.

[11] Hardik, B. K., \& Prabhu, S. V. (2017). Boiling pressure drop and local heat transfer distribution of helical coils with water at low pressure. International Journal of Thermal Sciences, 114, 44-63.

[12] Hardik, B. K., \& Prabhu, S. V. (2016). Boiling pressure drop and local heat transfer distribution of water in horizontal straight tubes at low pressure. International Journal of Thermal Sciences, 110, 65-82.

[13] Liao, Y. \& Lucas, D. (2016). Poly-disperse simulation of condensing steam-water flow inside a large vertical pipe. International Journal of Thermal Sciences, 104, 194-207.

[14] Nishikawara, M. \& Nagano, H. (2016). Numerical simulation of capillary evaporator with microgap in a loop heat pipe. International Journal of Thermal Sciences, 102, 39-46.

[15] Spinato, G., Borhani, N. \& Thome, J.R. (2016). Operational regimes in a closed loop pulsating heat pipe. International Journal of Thermal Sciences, 102, 78-88.

[16] Qu, J., Wang, Q. \& Sun, Q. (2016). Lower limit of internal diameter for oscillating heat pipes: A theoretical model. International Journal of Thermal Sciences, 110 174-185.

[17] Chung, T. J., Chen, J. W. \& Ferng, Y. M. (2016). Experimentally investigating heat transfer and boiling characteristics for the downward facing wall heating. International Journal of Thermal Sciences, 107, 96-104.

[18] Cai, E. Z. (2002). U.S. Patent No. 6,405,637. Washington, DC: U.S Patent and Trademark Office.

[19] Cai, E. Z. (2007). U.S. Patent No. 7,281,467. Washington, DC: U.S. Patent and Trademark Office.

[20] Aldridge, C. J., \& Fowler, A. C. (1992). Mathematical Modelling of Thermosyphons in Cryogenic Air Separation Plants. In Proceedings of the Sixth European Conference on Mathematics in Industry August 27-31, 1991 Limerick (pp. 75-78). Vieweg Teubner Verlag.

[21] Wallis, G. B. (1969). One-dimensional two-phase flow. McGraw-Hill Companies.

[22] LeVeque, R. J., \& Leveque, R. J. (1992). Numerical methods for conservation laws (Vol. 132). Basel: Birkhäuser. 